\title{
CUADROS DE MANDO Y SISTEMAS DE INDICADORES PARA LA GESTIÓN DE LOS DESTINOS TURÍSTICOS: UNA REFLEXIÓN DESDE LA SOCIOLOGÍA ${ }^{1}$.
}

\author{
MANUEL ÁNGEL SANTANA TURÉGANO ${ }^{2}$ \\ Departamento de Sociología y Antropología, Facultad de Economía, Empresa y \\ Turismo, Apartado 456, San Cristóbal de La Laguna- Tenerife. \\ NIEVES YANIRA HERNÁNDEZ FERNÁNDEZ ${ }^{3}$ \\ Cátedra de Turismo Cajacanarias- ASHOTEL, Facultad de Economía, Empresa \\ y Turismo, Apartado 456, San Cristóbal de La Laguna- Tenerife.
}

\begin{abstract}
Resumen: el objetivo de este trabajo es analizar la extensión de herramientas propias de la planificación estratégica de las empresas, como los cuadros de mandos y otros sistemas de indicadores, a la gestión de los destinos turísticos. Para ello el trabajo parte del análisis del surgimiento de los conceptos de "cuadros de mando" y de "cuadros de mandos integral", en el marco de la consolidación de la planificación estratégica como disciplina académica relativamente autónoma y como praxis profesional, dentro de la evolución de los sistemas empresariales en la segunda mitad del siglo XX. Posteriormente se hace un análisis de la aplicación de estos sistemas a la gestión turística, en primer lugar, en las empresas y posteriormente en la gestión de destinos turísticos. El trabajo concluye mostrando las limitaciones de este enfoque para la gestión de entidades socialmente tan complejas como son los destinos turísticos, debido a su carácter fuertemente organicista. Por ello, se propone como herramientas más efectivas el uso de metodologías, como las derivadas del denominado "Consenso de Saint Gallen", que permitan mejorar la gobernanza de los destinos.
\end{abstract}

Palabras clave: planificación estratégica, DMOs (organizaciones de gestión de destinos), gobernanza, Canarias, sostenibilidad, estudios de management críticos

\section{SCORECARDS AND INDICATORS SYSTEMS FOR THE MANAGEMENT OF TOURISM DESTINATIONS: A SOCIOLOGICAL APPROACH.}

\begin{abstract}
: the aim of this paper is to analyze the use of strategic management tools, such as scorecards and other indicators systems, to the management of tourism destinations. The paper starts by analyzing the rise of the concepts of scorecards and balanced scorecards within the broader field of strategic management as an autonomous academic field and a profession, in the second half of the XXth century. An analysis of how these concepts have been applied to the management of tourism firms and destinations follows. The paper concludes by showing the limitation of such an approach to manage so complex social institutions as tourism destinations, due to their organicist approach. Therefore, more useful methodologies, such as those stemming

\footnotetext{
${ }^{1}$ Este trabajo es resultado del proyecto "Sistema de Información para la medición de los impactos del turismo- SITUR", financiado a través de la Convocatoria de Ayudas a Proyectos de Investigación de la Fundación Cajacanarias, edición de 2016.

2 masantur@ull.es

3nyherfer@ull.edu.es
} 
from the so called "Saint Gallen consensus" are suggested in order to improve the governance of tourism destinations.

Keywords: strategic planning, Destination Management Organizations (DMOs), Governance, Canary Islands, Sustainability, Critic Management Studies.

\section{INTRODUCCIÓN}

El presente artículo surge de un proyecto más amplio que tiene como objetivo la elaboración de un sistema de indicadores para la medición de los impactos del turismo en Canarias, elaborado bajo el supuesto de que la aplicación práctica del conocimiento científico permitiría mejorar la gestión del sector turístico. Se partía por tanto del supuesto de que el desarrollo de un sistema de indicadores eficaz y eficiente, en forma de cuadro de mandos, permitiría mejorar la toma de decisiones y, en último término, favorecer el desarrollo económico y social provocado por el turismo, minimizando los impactos negativos que éste genera. El proyecto inicial, desarrollado desde un bagaje teórico propio de la Economía y Administración de Empresas, se basaba en los conceptos de cuadro de mandos y cuadro de mandos integral, que en la actualidad son considerados desde estas disciplinas como la manera más adecuada de gestionar empresas y organizaciones (Kaplan y Norton, 2000). La filosofía en que se basan estos sistemas de gestión sería que, si tenemos más y mejor información, podremos tomar mejores decisiones, y las buenas decisiones estratégicasserán las que permitirán que las empresas y destinos bien gestionados se adapten y prosperen, en un entorno de competitividad creciente. Por el contrario, las empresas y destinos que estén mal gestionados por carecer de sistemas de información adecuados acabarán perdiendo cuota de mercado, por lo que, en último término, disponer de sistemas de información eficaces y eficientes es un requisito indispensable para la supervivencia de las empresas y los destinos turísticos. La idea sería, en definitiva, que sólo las empresas y los destinos turísticos que se doten de buenos sistemas de información y de toma de decisiones acabarán sobreviviendo a medio largo plazo.

Sin embargo, estas premisas de partidachocaban con elenfoque sociológico y crítico desde el cual se pretendía desarrollar el proyecto.Por una parte, la idea de que la implementación de sistemas de información avanzados es clave para la adaptación al mercado puede resultar, en cierto sentido, contradictoria con la economía clásica, para la cual el mercado es el gran sistema de recogida de información. Por otra parte, la idea de que la adaptación de las empresas y organizaciones a su entorno es siempre el resultado de actuaciones intencionales y racionales de la dirección choca con buena parte de las aportaciones de la sociología de las organizaciones, que en la línea de los Critical Management Studies (véase, por ejemplo, Grey y Willmot, 2005), han señalado que las estrategias reales implementadas en las organizaciones son a menudo el resultado de equilibrios de poder contingentes, cuyas motivaciones están con frecuencia alejadas de las motivaciones explícitamente manifestadas. En cualquier caso, entendíamos que el diseño de un sistema de indicadores no podía concebirse como una cuestión técnica basada en una concepción del conocimiento científico que podría asimilarse a lo que se denominaría una investigación "confinada" o de laboratorio, siguiendo la terminología de Barthe, Callon y Lascoumes (2014), sino que parecía más útil un enfoque más cercano a lo que estos autores denominan "fórums híbridos", que combinara los saberes de profanos y especialistas. Para poder valorar en qué medida el desarrollo de sistemas de información más avanzados, que permitan inyectar más datos a los procesos de tomas de decisiones, podría mejorar la gestión de los destinos 
turísticos, este artículo analizará la aplicación decuadros de mando y cuadros de mandos integrales, herramientas derivadas dela planificación estratégica, entendida ésta como un campo de conocimiento técnico, a la gestión destinos turísticos. Para ello, en primer lugar, se analizará el surgimiento, desarrollo y extensión de los cuadros de mandos y cuadros de mandos integrales, y su aplicación al turismo. Posteriormente se enmarcarán este tipo de herramientas en la consolidación de la planificación estratégica como un conocimiento experto, un saber propio de especialistas, dentro del ámbito de conocimiento científico- técnico. A partir de aquí haremos un repaso de las dos corrientes de pensamiento más importantes en la gestión de los destinos turísticos, una más tecnocrática, ejemplificada en los manuales y recomendaciones elaboradas por organismos internacionales (enfoque top- down, de arriba hacia abajo), y otra más socio- política, centrada en las metodologías de consenso desarrolladas en torno al denominado "Consenso de St. Gallen" (enfoque bottom- up, de abajo arriba; véase Reinhold,Laesser, y Beritelli, 2015). La consideración del carácter socialmente complejo de los destinos turísticos nos llevará a plantear que las metodologías participativas, de abajo haciaarriba, siguiendo el modelo de investigación abierta, parecen tener un potencial mucho mayor para mejorar la gestión de los destinos turísticos que las de carácter técnico, top-down, que sigan un modelo de "investigación confinada".En definitiva, lo que pueden aportar los sistemas de información para la toma de decisiones no es una ayuda para encontrar "la respuesta correcta", dado que las cuestiones que atañen a gestión no admiten una única respuesta, por más que pretenda legitimarse ésta en base a una determinada manera de entender la Ciencia, sino, de manera más humilde, una ayuda para que un conjunto de actores, individuales o colectivos, encuentren "una respuesta" que, desde sus marcos interpretativos, sea, no ya "la correcta", sino simplemente satisfactoria o adecuada, lo que enlaza con los planteamientos de Simon (1991) sobre la "bounded rationality", retomados más recientemente por otros autores como Gigerenzer y Selten (2002) o Kahneman (2003). Pasamos a continuación a desarrollar estas cuestiones, y comenzaremos definiendo los conceptos a analizar, los de "cuadro de mando" y "cuadro de mandos integral".

\section{CUADROS DE MANDO: ORIGENES Y DEFINICIÓN.}

A punto de comenzar la segunda década del siglo XXI la elaboración de cuadros de mando o cuadros de mando integrales, como herramientas de recogida de información para la toma de decisiones, se han convertido en un elemento fundamental de la visión dominante acerca de cómo se han de gestionar las empresas y organizaciones. Una búsqueda del término "Balanced Scorecard", equivalente inglés del término español "Cuadro de Mandos Integral", en la base de datos Sciencedirect (www.sciencedirect.com), a finales de 2018, arroja más 4.300 resultados, de los cuales casi 800 corresponden a capítulos de libro y más de 2.700 a artículos de investigación. Por su parte, la base EBSCOHOST arroja más de 1.000 resultados para el mismo término, y la búsqueda en Google Académico del término registra más de 203.000 resultados, mientras que su traducción al español presenta en torno a 36.700 resultados. Fuera del ámbito académico, la búsqueda en Google por "balanced scorecard" arroja más de 20 millones de resultados, más de 700.000 el término español de "cuadro de mandos integral". El Cuadro de Mandos Integral se ha convertido no tan sólo en una herramienta de gestión que se enseña en Universidades y Escuelas de Negocio, sino también en un producto que es ofrecido como un servicio especializado, habiéndose convertido en uno de los más habituales en la consultoría de empresas y organizaciones. Existen multitud de empresas que ofrecen software para la realización de "cuadros de 
mandos integral", y numerosas consultorías, con y sin ánimo de lucro ${ }^{4}$ que se dedican, tal y como lo expresan en sus objetivos, "a ayudar a las organizaciones a lograr sus objetivos mediante la implantación de sistemas de Cuadros de Mando Integral". Dado que este tipo de herramientas son un producto cultural e histórico relativamente reciente, pues el propio término "Balanced Scorecard" no fue acuñado hasta 1992, y que obviamente en épocas anteriores las organizaciones encontrarían utilizaban otros medios para lograr alcanzar sus objetivos, ¿de dónde surge el concepto? ¿Cómo ha llegado a alcanzar la importancia que tiene actualmente?

El término de cuadro de mando, como herramienta de gestión empresarial, viene siendo utilizado de forma habitual desde hace ya casi medio siglo, aunque sus orígenes son aún más antiguos. En la década de 1930 ingenieros franceses desarrollaron el "tableau de bord", precursor del cuadro de mandos, con el objetivo de medir los avances en los procesos de producción (Meyer, 1969). En las décadas de 1950- 1960 los cambios en las formas de organización empresarial subrayan la necesidad de definir y establecer lo que se consideraban indicadores objetivos que resultan claves en el éxito empresarial, lo que llevó al desarrollo de los cuadros de mando, como muestran los trabajos realizados por Lauzel, Cibert y Carrió (1967). Aunque ya por entonces a los indicadores meramente productivos desarrollados por los ingenieros en los años 30 se habían añadido otros indicadores de carácter presupuestario o financiero, hasta la década de los 80 se pensaba en un cuadro de mandos fundamentalmente como un informe que permitía comparar los resultados reales de la actividad empresarial con los inicialmente planteados, y podía permitir, en consecuencia, realizar las acciones de corrección oportunas (Russo, 2005). Durante la segunda mitad del siglo XX el cuadro de mandos se fue generalizando entre algunas de las empresas más influyentes del mundo. Así, por ejemplo, General Electric lo utilizaba como instrumento de gestión para llevar a cabo el seguimiento de los procesos, incorporando indicadores que controlaran la consecución de los objetivos, a corto y a largo plazo (véase Dávila, 1999). En el ámbito de habla hispana un importante hito en la difusión del cuadro de mandos fue la propuesta de Blanco, que presentó en su libro una propuesta de cuadro de mando en el que se incluían gráficos y tablas, que denominó "banco de datos" (Blanco, 1976:104).

Con el transcurrir de las décadas el Cuadro de Mando (CM) pasó de ser un informe con indicadores para el control financiero de la empresa a incluir indicadores de materia no financiera (Dávila, 1999), y se acaba concibiendo como una herramienta fundamental en el trabajo de los directivos. De manera que, en formulación de Escobar, el cuadro de mando se acaba consolidando como "un mecanismo ideal para canalizar gran parte de la información contable que demandan los directivos, adoptando el concepto de información necesaria y suficiente presentada en un formato de fácil lectura y rápido uso como la piedra angular sobre la que se ha de cimentar el desarrollo del sistema de información contable" (Escobar Rodríguez, 1999: 1075). El cuadro de mandos es visto como útil en la medida en que ofrece una información sencilla, sinóptica y eficaz para el proceso de toma de decisiones, pero pese a su innegable utilidad (Graner, 1991) el cuadro de mandos tradicional presentaba limitaciones, ya que, por ejemplo, carecía de una perspectiva de integración que reflejara la visión que se tenía de la empresa, y además no establecía una relación clara entre los indicadores incluidos (Santos y Fidalgo, 2005).

\footnotetext{
${ }^{4}$ Existe por ejemplo el "The Balanced Scorecard Institute", véase https://www.balancedscorecard.org/
} 
Dado que los cuadros de mando no permitían una visión estratégica de la empresa, ya que si bien ayudaban a identificar factores claves no permitían a las organizaciones tener una visión clara de las causas y efectos que influyen en los elementos claves de su éxito, a partir de la década de 1990 se da el paso de los "Cuadros de Mandos" a los "Cuadros de Mandos Integrales" (Balanced Scorecard). La falta de integración de los cuadros de mandos tradicionales con lo que se entendía que era la misión de la empresa, así como el que la elección de los indicadores la realizara el directivo, teniendo como base su propia intuición y experiencia (Dávila, 1999), originó que, a principios de los años 90, R. Kaplan, profesor de la Harvard Business School, y P. Norton, consultor empresarial, presentaran una nueva propuesta que pretendía superar estas limitaciones: el Cuadro de Mandos Integral, (CMI), traducción del inglés Balanced Scorecard (BSC). El concepto, aparecido en el número de enero-febrero de 1992 de la Harvard Business Review (Kaplan y Norton, 1992), se basó en un estudio realizando en doce empresas, elegidas en función de cómo medían su rendimiento, y planteaba que los indicadores de carácter financiero deberían ser complementados con indicadores de carácter no financiero. Por todo ello el Cuadro de Mandos Integral (CMI) puede considerarse como un refinamiento del cuadro de mandos clásico que traduce la estrategia y la misión de una organización en un conjunto de indicadores que informan de la consecución de los objetivos.

En definitiva, la principal diferencia entre un cuadro de mandos y un cuadro de mandos integral (CMI) es que mientras que el primero consiste en una serie de indicadores que dan información sobre la marcha de la organización, un verdadero cuadro de mandos integral debería ser "un conjunto de indicadores que proporcionan a la alta dirección una visión comprensiva del negocio" (Kaplan y Norton, 1992:71, traducción propia). O, dicho de otra forma, el cuadro de mandos se puede hacer sin tener en cuenta la estrategia de la organización, bajo el supuesto de que los indicadores que en él se incluyen son fundamentales para medir el desempeño, independientemente de la visión estratégica, mientras que el cuadro de mandos integral parte de la idea de que los indicadores sólo tienen sentido en función de la estrategia: en función de cuál sea ésta, será necesario recabar información sobre unas u otras cuestiones. El cuadro de mandos integral, en cuanto que sistema de indicadores para obtener una visión comprensiva del negocio se organiza en torno a cuatro perspectivas, que responden a cuatro cuestiones fundamentales en toda empresa u organización: 1. Perspectiva de los clientes: ¿cómo nos ven los clientes?; 2. Perspectiva financiera: ¿cómo nos ven los accionistas?; 3. Perspectiva interna del negocio: ¿en qué debemos sobresalir?) y 4. Perspectiva de la Innovación y aprendizaje:¿cómo podemos seguir mejorando y creando valor? (Kaplan y Norton, 1992). Tras la publicación de su artículo, Kaplan y Norton desarrollaron su idea de los CMI en obras posteriores, reclasificando dos de las cuatro perspectivas definidas inicialmente (Kaplan y Norton, 1996). Con el desarrollo del concepto de cuadro de mandos integral (CMI), éste se acabó viendo como un mapa de la estrategia empresarial, en el que las cuatro perspectivas se integran con los objetivos de la empresa, con el fin de visualizar qué habría que hacer para obtener los resultados esperados en función de la estrategia (Kaplan yNorton, 2004), de manera que "un buen BSC debe reflejar el mapa estratégico del negocio diseñado por la gerencia para comunicar e implementar la estrategia" (Ballvé, 2006: 17).En definitiva, el desarrollo de un CMI implica formular una estrategia, comunicarla, establecer indicadores de cumplimiento y hacer un seguimiento de en qué medida la gestión está logrando los objetivos inicialmente planteados, proponiendo medidas correctoras en caso de que esto 
no se lograra. En virtud de ello, el CMI podría considerarse como parte del giro humanista en la gestión, ya que de alguna manera amplía el ámbito de la gestión más allá de lo meramente financiero hasta las cuatro perspectivas anteriormente mencionadas. Además, el CMI pretende convertirse en un elemento de retroalimentación que permita adaptar las decisiones empresarialesa lo que efectivamente está sucediendo en cada momento, en función de la visión estratégica de la dirección, facilitando así las constantes adaptaciones de la estrategia empresarial al entorno tan cambiante en el que operan en la actualidad las organizaciones. (Mora y Vivas, 2001).

\section{LA DIFUSIÓN DE LOS CUADROS DE MANDO.}

¿Cómo ha llegado a convertirse el uso de Cuadros de Mando Integrales (CMI) en la nueva ortodoxia de la gestión de empresas y organizaciones? De acuerdo a uno de los autores más influyentes en el campo, Henry Mintzberg, las prácticas y los estilos en la gestión de las organizaciones (management) puede sintetizarse en torno a un triángulo de posiciones. Para unos, la gestión puede entenderse como un oficio o un tipo especial de artesanía (craft), que involucra sobre todo a la experiencia y al aprendizaje práctico. Para otros, la gestión (management) es un arte, y atañe a cuestiones como la visión y las intuiciones creativas. Por último, para un tercer grupo de posiciones la gestión sería una ciencia, y tendría que ver sobre todo con el análisis y la recopilación sistemática de evidencias (Mintzberg, 2004). En los últimos años se ha vuelto cada vez más dominante la visión de la gestión como una ciencia (Scientific Management), lo que tiene que ver con el prestigio social creciente de la Ciencia y la Tecnología, por lo que comenzaremos nuestro análisis de la difusión de los cuadros de mando como visión dominante de la gestión moderna como si se tratara de una teoría científica.Desde una visión un tanto simple de lo que son las teorías científicas, que debería sin duda matizarse por aportaciones clave como la de Kuhn (véase Kuhn, 2004) acerca de las revoluciones científicas, se podría plantear que unos paradigmas científicos se imponen a otros sencillamente porque son capaces de explicar mejor la realidad. Siesto sucede en el terreno de la ciencia pura, en el de la ingeniería (ciencia aplicada) se supone que los nuevos enfoques y desarrollos derivarían de la aplicación de nuevos paradigmas científicos para intervenir y transformar la realidad.Desde esta visión que podríamos considerar propia de un positivismo un tanto ingenuo, los avances científicos, transformados en tecnología, acaban incidiendo y, desde una visión optimista, generalmente mejorando, la vida cotidiana de las personas. En los terrenos típicamente propios de la ingeniería cabría pensar en múltiples ejemplos en que esto efectivamente ha sido así, y han permitido la consolidación de avances tecnológicos que hoy forman parte de nuestra vida cotidiana.

Este tipo de argumentos se utiliza a menudo para justificar la difusión de innovaciones organizativas como los cuadros de mandos integrales. En este caso, las Ciencias Sociales harían "descubrimientos" en el ámbito de cómo funcionan las organizaciones, y propuestas organizativas como las analizadas serían la aplicación práctica, ingenieril, de las mismas. Continuando en esta línea de razonamientola lógica del cuadro de mandos se acabaría imponiendo porque, al ser una teoría "mejor" produciría "organizaciones mejores", y en la lucha por la supervivencia, en un mercado competitivo, sólo las mejores organizaciones acabarían sobreviviendo, y las que utilizaran métodos poco prácticos (poco científicos) se verían abocadas a su desaparición. Con lo cual, la respuesta a la cuestión con la que iniciamos este apartado 
sería que si los cuadros de mandos integrales se han convertido en la nueva ortodoxia de la gestión de las organizaciones ha sido porque son el sistema organizativo que ha permitido una mejor gestión de las organizaciones, por lo que, desarrollados inicialmente por tan sólo unas pocas organizaciones pioneras, han sido copiados, con mayor o menor acierto, por el resto de organizaciones para alcanzar unos umbrales mínimos de eficiencia que les han permitido su subsistencia.

La lógica de los cuadros de mando pretende, mediante un esclarecimiento de los factores causales que llevan a las organizaciones a alcanzar sus objetivos, utilizar el conocimiento "científico" acerca de lo que se considera que ha producido (en el pasado) el éxito de algunas organizaciones para producir, en el futuro, el éxito de las organizaciones así dirigidas. Pero, dado que las organizaciones se desenvuelven en entornos que pueden considerarse sistemas complejos (siguiendo la terminología de Lorenz, véase Silver, 2012) en que las acciones de unos agentes pueden influir en las de otros de manera que es prácticamente imposible de predecir, la difusión de sistemas de gestión como los "cuadros de mandos integrales" no puede explicarse completamente en base a estas ideas. Aún es más: este argumento tiene una importante debilidad lógica. Si la fórmula del éxito empresarial u organizativo pudiera deducirse del conocimiento "científico" disponible en los medios especializados los fracasos serían tan sólo el resultado de una negativa a aplicar el conocimiento. Pero dado que en un entorno competitivo el fracaso de algunos es conditio sine qua non para el éxito de otros, y dado que el conocimiento es relativamente accesible, hay más fracasos que los que se derivarían de una mera negativa a adoptar las formas de gestión más modernas. $\mathrm{O}$, dicho de forma mucho más concreta: en un entorno en que todas las empresas y organizaciones usan metodologías basadas en los cuadros de mandos, no todas triunfan o, cuando menos, unas triunfan más que otras, con lo que no puede afirmarse que el uso de cuadro de mandos sea, per se, el principal factor que explica el éxito de una organización. Por ello, la extensión de los cuadros de mando no se puede entender adecuadamente aludiendo tan sólo a esta idea propia de lo que a menudo se denomina "ecología organizacional". Es por tanto necesario contemplar, siguiendo las ideas de Mintzberg (2004) a la gestión también como un arte y como un oficio artesano.

Para entender el auge de los cuadros de mando integral como elemento clave de las formas de organización contemporáneas partiremos aquí desde una perspectiva crítica, basada en los postulados de la Sociología Económica. Partiendo desde una visión crítica de la Economía, en la que el poder es un elemento central en todas las relaciones económicas, que determinadas organizaciones tengan mejores capacidades de predecir el futuro no tiene que ver tanto con que hayan desarrollado sistemas técnicos más perfeccionados, sino con que su poder de mercado les permite, más que predecir, preformar el futuro. Para un pequeño hotel independiente, no perteneciente a una cadena, el futuro puede ser totalmente impredecible, como, por ejemplo, si a un año visto los clientes van a aumentar o disminuir. Por el contrario, para un tour operador importante, que no sólo canaliza la demanda turística, sino que en buena parte la crea, la misma cuestión puede ser completamente predecible. Por ello, pese a la pretensión de algunos de convertir a la disciplina en una suerte de ingeniería, planificación estratégica es un caso paradigmático de lo que autores como Callon (2010) denominan la performatividad de la economía: la disciplina no trata tanto tan sólo de describir cómo son las cosas, sino también prescribe cómo deberían ser. La teoría de la organización performa, es decir, da forma, a la realidad que supuestamente tan sólo pretendía analizar de manera aséptica y científica. Siguiendo el enfoque de Fernández Rodríguez (2007), y anteriormente, de Boltanski y Chiapello (2002), los manuales de gestión empresarial 
pueden entenderse como un estilo literario, que se difunden por la acción favorable de determinados actores clave, con poder e influencias, de manera similar a la que se difunden los estilos literarios. Por ello pasamos a continuación a tratar la consolidación de la planificación estratégica, y dentro de ella el enfoque de los cuadros de mando, como campo de conocimiento experto.

\section{LA PLANIFICACIÓN ESTRATÉGICA COMO CONOCIMIENTO EXPERTO.}

El surgimiento de la planificación estratégica fue una respuesta a los desafíos que el crecimiento empresarial y el desarrollo del capitalismo planteó a las grandes empresas transnacionales, principalmente norteamericanas a partir de la Segunda Guerra Mundial. La organización del trabajo ha constituido, desde los inicios de la Revolución Industrial, una cuestión social clave y tiene un componente técnico, ya que, para un estado dado del avance tecnológico, y un sector productivo concreto se puede pensar que hay una manera más eficaz y eficiente de organizar la producción, lo que no es, en último término, más que la idea de la "one best way" de Taylor. Los métodos tayloriano fordistas, desarrollados durante la primera mitad del siglo XX, constituyeron una solución tecnológica a la cuestión de cómo maximizar la producción, que fue relativamente independiente del contexto social, entre otros motivos porque el objetivo de maximizar la producción no estaba en cuestión. Pero la organización del trabajo, ligada al reparto de la producción, no es una cuestión que tenga sólo un componente técnico, sino que tiene también un componente socio- político, pues sólo es posible dentro de los límites que resultan socialmente aceptables en un contexto históricogeográfico determinado. Como han establecido diversos estudios clásicos de la sociología del trabajo es necesario crear un mínimo consenso, logrando que los obreros acepten realizar las tareas diseñadas por los ingenieros, para garantizar que soluciones "eficaces y eficientes" en el mundo ideal de los técnicos no se transformen, al intentar llevarlas a la realidad, en utopías teóricas pero ineficaces e ineficientes en la práctica. El capitalismo industrial de finales del XIX y la primera mitad del XX, al concentrar a los trabajadores en un único lugar, permitía a la dirección un control directo de la producción. Pero también, al concentrar a los trabajadores, creaba las condiciones para el surgimiento de la acción colectiva, y la lucha obrera podía paralizar la producción con relativa facilidad. Para soslayar esta dificultad había dos vías posibles, una políticoideológica y otra técnica. Por la vía político- ideológica el capitalismo burocrático del XIX había encontrado un importante aliado en el surgimiento de una "ética del capitalismo", al estilo de la que estudiara Weber. A partir de la segunda mitad del siglo XX, como ilustran, entre otros, Boltanski y Chiapello (2002) diversos cambios tanto en los valores de las sociedades como en la organización económica de las mismas hacen necesaria la emergencia de una "cultura del nuevo capitalismo", en la formulación deSennett (2006). Las formas organizativas contemporáneas, facilitadas por las nuevas tecnologías, vienen condicionadas en gran medida por las posibilidades de controlar y ejercer el poder.

Éste el contexto amplio en el que paulatinamente, a partir de las décadas de 1950 y 1960, surge la disciplina de la planificación estratégica, o "estrategia de negocios", como traducción del concepto anglosajón de "Strategic Management", a partir de la obra de teóricos y académicos pioneros como Drucker, Selznick, Chandler o Ansoff, con bagajes teóricos bastante heterogéneos, y posteriormente desarrollada y consolidada 
por autores como Henry Mintzberg y Michel Porter ${ }^{5}$. La planificación estratégica podría definirse como "un proceso sistemático de desarrollo e implementación de planes para alcanzar fines, que implica el establecimiento de objetivos claros y cuantificables, de cara a poder evaluar en qué medida la planificación alcanza finalmente los objetivos inicialmente planteados". Dentro de esta definición general de la planificación estratégica cabe diferenciar distintas corrientes, conceptos y teorías, sin que exista acuerdo unánime acerca de los términos a utilizar. Entre las corrientes más destacadas cabe mencionar el análisis de Debilidades, Amenazas, Fortalezas y Oportunidades (DAFO o SWOT, por sus siglas en inglés), el análisis de los factores políticos, económicos, sociales y tecnológicos (análisis PEST), la utilización de las cinco fuerzas de la competitividad de Porter para analizar la planificación o el uso de cuadros de mandos integrales.

En la actualidad la planificación estratégica se ha convertido en habitual entre los gestores de empresa, y asignaturas con el título específico de "planificación estratégica" se imparten en muchas universidades de habla hispana, y cuando no existen asignaturas con ese título exacto conceptos relacionados con la planificación estratégica se imparten en otras asignaturas. Para el sector que aquí analizamos, el turismo, es digno de mencionar que existen asignaturas de Planificación o Dirección Estratégica del Turismo en los grados en Turismo que se imparten en muchas universidades españolas. Además, muchas universidades imparten títulos de máster oficial en turismo bajo el título de "Máster en Dirección y Planificación del Turismo". ¿Cómo de un contexto original de Guerra Fría en que la mera mención de "planificación" parecía incompatible con la idea de la "economía capitalista" (la economía planificada era la de los países llamados "socialistas") hemos pasado a la situación actual en que se tiende a asociar economía y planificación estratégica?La importancia alcanzada por la planificación estratégica, entendida como un campo en el que sólo las personas con conocimiento deberían de tener capacidad para tomar decisiones, se relaciona con la importancia que se da en las sociedades contemporáneas a los sistemas de conocimiento técnicoexperto, basados (al menos supuestamente) en el conocimiento científico. Desde la conformación de las sociedades modernas occidentales, a partir de las revoluciones de los siglos XVIII y XIX el conocimiento experto basado en la ciencia ha sido el criterio a partir del cual se ha asumido que debería organizarse la sociedad. El ideal positivista era el gobierno de los tecnócratas, ingenieros sociales que, de la misma manera que los ingenieros naturales aplicaban el conocimiento de las ciencias de la naturaleza para el dominio y la domesticación de la misma, aplicaran el conocimiento de las ciencias de la sociedad para una mejor gestión de ésta. La versión actual de este fenómeno se manifiesta en lo que se ha dado en llamar "Econocracia" (Earle, Moran, y Ward-

${ }^{5}$ Drucker, nacido en Austria, siendo su padre un funcionario de alto nivel entró en contacto en su casa con las ideas de Schumpeter, Hayek o Mises, según se cuenta en la web de la "Drucker Society" visitantes habituales de la misma (http://www.druckersociety.at/index.php/peterdruckerhome/biography). Obtuvo un doctorado en Derecho Internacional en la Universidad Goethe de Frankfurt en 1931, y tras trasladarse a los Estados Unidos a finales de dicha década fue profesor de Management entre 1950 y 1976, habiendo desarrollado en California los primeros MBA (Master in Business Administration), hoy tan imitados por todo el mundo. Selznick fue profesor de Sociología en Berkeley, mientras que Chandler puede considerarse un historiador económico que impartió sus enseñanzas en lugares tan prestigiosos como el MIT, la John Hopkins University o la Harvard Business School. Igor Ansoff, por su parte, formado en física y matemática, fue profesor en UCLA, aunque quizá ejerció mayor influencia en su calidad de consultor para multinacionales como Philips, General Electric o IBM. Por último, entre los autores más recientes, tanto Mintzberg como Porter tuvieron formación inicial en ingeniería, igual que Frederick W. Taylor, el ingeniero que transformó la organización industrial de finales del XIX y primera mitad del XX 
Perkins, 2016): el gobierno de las sociedades contemporáneas se justifica aludiendo a la "Economía", entendida ésta como una ciencia positiva que permite gestionar las sociedades de una manera más eficiente, y la práctica totalidad de las decisiones son tomadas por personas e instituciones que, o bien tienen formación en economía y administración de empresas (management) o bien tienden a justificar sus decisiones en términos y argumentos propios de estas disciplinas.

Que la opinión de algunas personas deba pesar más que la de otras a la hora de tomar decisiones se fundamenta en la idea de que, al menos en los campos del conocimiento técnico o experto, no todas las opiniones valen lo mismo. En nuestras sociedades se tiende a pensar que las opiniones de quienes tienen un mejor conocimiento científico- técnico de la realidad son mejores que las de los profanos, y por lo tanto los técnicos han de tener un rol preferente a la hora de tomar decisiones y diseñar políticas. Desde mediados del siglo XX, y de manera especialmente acentuada desde la década de 1980, el ámbito económico se ha venido considerando como un ámbito propio del conocimiento experto, en el cual las decisiones deberían ser tomadas por quienes tienen conocimientos avanzados de economía. ¿En qué medida existe un ámbito de conocimiento experto económico, que justifica que se dé más importancia a las opiniones de las personas que lo poseen a la hora de organizar la economía y la sociedad? ¿Qué diferencia aquellos ámbitos en que cabe hablar de conocimiento experto y aquellos en que no? Siguiendo ideas sugeridas por Latour (1992) plantearemos aquí la diferenciación entre ámbitos de conocimiento experto- técnico y ámbitos de conocimiento socio- político como polos extremos de un continuo de posiciones. Serían cuestiones propias del conocimiento experto técnico aquellas en las cuales la evolución de los fenómenos es independiente de cómo se formulen las cuestiones, de quiénes las formulen y de las medidas que se adopten para intervenir. De acuerdo con la clásica distinción de Weber, se trata de ámbitos en que predomina lo que él denominaba: racionalidad instrumental, medios- fines o económica. Así, los ingenieros asocian materiales para construir puentes, y los médicos utilizan tratamientos y medicinas para obtener unos fines, como pueden ser el preservar la vida humana que les son dados y que no deciden por sí mismos. En estos ámbitos no todas las opiniones valen lo mismo, sino que se otorga más valor a la persona con mayor conocimiento técnico. El conocimiento experto- técnico es acumulable, pues cada vez que alguien resuelve una cuestión ésta queda resuelta para siempre. Por contraposición a este conocimiento experto técnico, en el extremo opuesto del conjunto de posiciones nos encontraríamos con que serían cuestiones propias del conocimiento socio- político aquellas en las cuales la evolución de los fenómenos depende de cómo éstos se formulen, de las personas que realicen la definición de los mismos y de las propuestas que hacen para intervenir en la realidad.En estos campos no cabe hablar de acumulación del conocimiento: la cuestión de qué es una sociedad justa y cómo debe de organizarse, que ocupan a los filósofos desde la Antigüedad, son preguntas que nunca pueden darse por resueltas y que cada sociedad humana que exista deberá de responder, siempre en relación con sus valores.

Hecha esta distinción en términos de tipos ideales, cabe plantear que las cuestiones que atañen a la planificación estratégica están más cercanas al polo del conocimiento socio- político que al del experto- técnico, si bien han conseguido buena parte de su prestigio social auto presentándose como un ámbito de conocimiento experto. A nivel micro podría pensarse que la disciplina se ocupa de cuestiones relativas a cómo garantizar que una organización alcance sus objetivos de manera eficaz y eficiente, lo que podría parecer, a primera vista, un ámbito propio del conocimiento 
experto. Pero lo cierto es que estas cuestiones sólo tienen respuesta considerando a los actores implicados: ¿es más eficiente una empresa que genera más beneficios empresariales o más y mejores salarios? Además, la cuestión de la eficiencia cambia si consideramos los costes ambientales o no, y además se trata de cuestiones para las cuales no cabe una acumulación del conocimiento de manera que una vez respuesta una cuestión quede respuesta para siempre. Históricamente han existido diversas respuestas a preguntas como "cuál es la mejor forma de organizar una empresa u organización" o "qué impacto tiene el turismo en las sociedades receptoras". A nivel macro, la planificación estratégica, trata cuestiones claramente políticos, pues en la medida en que se ocupa de medios para alcanzar objetivos, resulta imposible separar los modos de alcanzar y medir el cumplimiento de objetivos de los modos de definir cuáles sean éstos.

En cualquier caso, en este trabajo se parte de la idea de que, en la práctica, la respuesta a cualquier cuestión implica tanto al conocimiento técnico experto como al político- estratégico. Partiendo de la perspectiva de los estudios sociales de la ciencia (SSS, Social Studies of Science) se plantea que en realidad ninguna cuestión es meramente técnica, sino que mediante distintos procesos las cuestiones técnicocientíficas y político- sociales se encuentran en la práctica intrínsecamente entrelazadas, de manera que en la mayoría de los casos es imposible separarlas. Siguiendo el trabajo de Barthes, Callon y Lascoumes, (2009) lo que haremos es seguir lo que estos autores denominan procesos de traducción, que acaban transformando lo que son incertidumbres o cuestiones en el ámbito de la ciencia en respuestas, que aparentan aportar certidumbre, en el ámbito de la política, mediante lo que se logra, según lo denominan estos autores, el cierre de las controversias. La filosofía del cuadro de mandos integral implica establecer a priori relaciones causales, del tipo "si $A \rightarrow B$ ", que permitan identificar cuáles son los aspectos claves en los cuales una organización se ha de centrar para conseguir sus objetivos. Así por ejemplo, "si mis clientes están satisfechos $\rightarrow$ mejoraré mi cuenta de resultados". Este tipo de relaciones permite, en una segunda fase, establecer indicadores que hacen posible cuantificar en qué medida se están obteniendo los objetivos que inicialmente se pretendían. Aparentemente se trata de un tipo de razonamiento típicamente instrumental: no entra en valoraciones acerca de si los objetivos de una organización son más o menos deseables, sino tan sólo trata de ver si se están logrando. Pero lo cierto es que el mero hecho de ponerse a pensar en indicadores acerca de cómo medir el cumplimiento de los objetivos de una organización implica una aceptación ética de los mismos. Poniendo un ejemplo del sector turístico: se suele considerar el número de turistas que llegan a un destino como un "KeyPerfomance Indicator". Desde un punto de vista conservacionista podría pensarse que un número más elevado de turistas es "malo": en aras de preservar la pureza medioambiental o cultural de un destino, o incluso de mantener elevado su precio, sería deseable un menor número de turistas. Pero también podría argumentarse, desde el punto de vista contrario, que un número de turistas más elevado es bueno porque implica, por ejemplo, mayores beneficios económicos para el destino, y puede ayudar a preservar el patrimonio natural y cultural. La creación de un "cuadro de mandos integral" implica establecer qué se va a medir (el número de turistas) y además una determinada manera de interpretar los resultados (que los turistas suban ¿es buen o es malo?) Y este marco interpretativo pretende fundamentarse en lo que se entiende que es el conocimiento científico: en turismo se viene hablando desde hace cierto tiempo del concepto de "capacidad de carga" término que se asocia frecuentemente a la ecología: si podemos establecer, en base al conocimiento científico la capacidad de carga de un destino, podremos intentar 
alcanzar un número de turistas que maximice los beneficios para el destino sin provocar un perjuicio al medioambiente, a la cultura y la sociedad local.

En definitiva, ¿cómo se traduce lo que en el ámbito científico son preguntas en respuestas que guíen la elaboración de estrategias y políticas? Lo que Barthes, Callon y Lascoumes (2014) denominan "el cierre de las controversias" se hace a partir de cómo van evolucionando los debates académicos y científicos. La concepción de la planificación estratégica del turismo como un campo de conocimiento experto implica que se asuma el supuesto de que el conocimiento es acumulable. Por ello, copiando los modos de actuación de las ciencias naturales, se tiende a pensar que es posible la acumulación del conocimiento sobre la materia, lo que lleva a la utilización de lo publicado en revistas consideradas "de impacto" como saberes contrastados e incontestables que deberían de guiar la gestión. Los últimos conocimientos científicos, según se exponen en las revistas de impacto, permiten responder a cuestiones del tipo de la mencionada anteriormente "qué hace que los clientes estén satisfechos" lo que permitiría convertir la planificación estratégica en una cuestión meramente técnica: aplicar los conocimientos científicos permite elaborar recetas que permiten alcanzar unos objetivos dados de una manera mecánica y automática. Por ejemplo: 1) "De acuerdo a lo publicado en "Journal of International Tourism", si controlo los factores $A, B, C \rightarrow$ mis turistas estarán satisfechos 2) Si mis turistas están satisfechos $\rightarrow$ lograré mis objetivos 3) Ergo, para lograr mis objetivos tengo que controlar los factores A, B, $C$, y por lo tanto elaborar indicadores para medirlos. La globalización de las universidades y de los servicios de consultoría contribuyen a generar consenso en las prácticas de la planificación estratégica del turismo, que se centran en la idea de que existen tres grandes campos de análisis en la medición y gestión del turismo: el impacto económico, el impacto cultural y el impacto medio- ambiental, que deben y pueden considerarse de manera autónoma, buscando indicadores para cada una de éstas áreas (un enfoque alternativo, por ejemplo, sería el de indicadores que tuvieran en cuenta la relación entre las distintas áreas más que el desempeño de cada una de éstas por separado). De la misma manera que una revisión del "estado de la cuestión" acerca de la fabricación de microchips, por ejemplo, nos pondría al día de cuáles son los métodos actualmente más avanzados para fabricar microchips, una revisión de la literatura científica sobre el turismo nos debería de permitir acumular conocimiento acerca de cómo medir y gestionar mejor un destino turístico. Y la revisión de la literatura parece señalar que la gestión de los destinos turísticos podría mejorarse si se recolectara más y mejor información, en forma de indicadores, sobre los impactos económicos, sociales y medio ambientales del turismo.

¿Qué se considera un indicador turístico? La organización de referencia en la materia, la OMT (Organización Mundial del Turismo) define un indicador como "un instrumento cuantitativo, sintético, que facilita el análisis y la evaluación de la información de manera tal que, al ser utilizado en combinación con otro tipo de instrumentos, evita a los tomadores de decisiones la posibilidad de tomar, de manera involuntaria, malas decisiones" (extraído de Torres y Palomeque, 2014, traducción y adaptación propia). Esta definición, pese a presentarse en términos propios de ámbitos de conocimiento experto- técnico, remite a cuestiones subjetivas, como la de qué es una mala decisión, que claramente pertenecen al ámbito de conocimiento socio- político. Que una decisión sea buena o mala dependerá, en primer lugar (1), de los actores 
implicados en la cuestión ${ }^{6}$. En segundo lugar, (2) la respuesta a la pregunta sobre "qué es una mala decisión" dependerá de cómo se defina la cuestión ${ }^{7}$. Y, en tercer lugar (3) ¿cómo separar lo que es una mala decisión de las propuestas concretas de intervención? En definitiva, lo que estamos planteando es que la consolidación de métodos como los cuadros de mando como herramientas para la planificación estratégica tiene que ver con que ayuda a presentar el área como un ámbito en que es posible la acumulación de conocimiento técnico, y al hacer esto contribuye a dotar de legitimidad a determinadas propuestas, que se tienden a auto presentar como "científicas", y a desprestigiar a otras propuestas, que se tienden a presentar como ideológicas y contrarias a la ciencia.

\section{PLANIFICACIÓN ESTRATÉGICA DEL TURISMO.}

Siguiendo esta distinción que acabamos de realizar, la literatura sobre la planificación estratégica del turismo puede dividirse en dos grandes polos en función de que conciban la planificación estratégica como un campo de conocimiento científico o experto o como un ámbito de conocimiento más cercano al arte o a la artesanía, siguiendo la terminología de Mintzberg (2004). Desde un punto de vista que concibe a la planificación estratégica como una cuestión científico-técnica, la relativamente abundante literatura sobre la medición de los impactos del turismo y el uso de instrumentos de carácter cuantitativo para la gestión del mismo suele partir de lo actualmente existente para realizar una propuesta de indicadores que se intenta validar mediante un cuestionario. Así por ejemplo, la propuesta de Torres Delgado y Palomeque (2014) parte de los tres ámbitos sobre los que parece haber consenso en los impactos del turismo: una dimensión sociocultural, una dimensión económica y una dimensión ambiental, identifica los factores que causan la presión y llega por último a una propuesta concreta de indicadores que se concreta en la medición de aspectos como inversión pública o en turismo, población residente y espacios y establecimientos turísticos con certificación medio- ambiental. Por su parte, el trabajo de Vila et al (2010), uno de los pocos que específicamente utiliza el término "balanced scorecard" en su aplicación al turismo, identifica indicadores como nivel de precios, satisfacción de los residentes, temporalidad o nivel de ingresos de la población local. El artículo de Torres Lorenzo y Hernández Martín (2013) utiliza indicadores como llegada de turistas, plazas totales ofertadas, total de empleos registrados o empresas inscritas en la Seguridad Social. La Unión Europea, en su "Sistema Europeo de Indicadores Turísticos" (EU, 2010) hace una propuesta basada en 43 indicadores bases y otros complementarios. A diferencia de enfoques más tradicionales, que suelen dividir el tema en tres áreas, agrupa los indicadores en cuatro grandes áreas, pues añade una primera área "Gestión del destino" (Destination Management) a las tres tradicionales: valor económico (Economic Value) impacto social y cultural e impacto medio ambiental, concretándose en indicadores muy específicos, como pueden ser, por ejemplo, estancia media y tasa de ocupación en alojamientos, que se consideran indicadores del rendimiento de las empresas turísticas, que vendría a ser una dimensión del impacto económico.

\footnotetext{
${ }^{6}$ Un conservacionista considerará buena decisión aquella que minimiza el impacto del turismo en un territorio, mientras que un desarrollista pensará que es buena la que maximiza el impacto económico ${ }^{7}$ Por ejemplo, y volviendo a ejemplos anteriores: ¿es una buena decisión desde el punto de vista ambiental aquella que no ocasiona más daño al ecosistema, partiendo desde el punto actual del desarrollo turístico? $¿ \mathrm{O}$ es buena la medida que permite al ecosistema recuperar condiciones previas a los daños ambientales infringidos por el turismo?
} 
Pero existe también otra literatura sobre la planificación estratégica del turismo que tiende a concebirla como una tarea social, ejemplificada en la idea del "Consenso de St. Gallen" (véase, Laesser y Beritelli, 2013) cuyo enfoque, más cualitativo, es considerablemente distinto. Según (Laesser y Beritelli, 2013, traducción propia), la toma de decisiones mediante consenso (consensus decision making) se definiría como "un proceso grupal que busca la aprobación, aunque no necesariamente el acuerdo, de los participantes en temas clave, y la resolución de las objeciones", partiendo de la doble definición del término consenso como "acuerdo generalizado" y "solidaridad grupal de sentimientos y creencias". A partir de las reuniones del "Forum on Advances in Destination Management", celebrado cada dos años desde 2012, la metodología para convertir los conocimientos "teóricos" en saberes prácticos para la gestión de los destinos turísticos pasa por una metodología en cinco etapas que parte de la identificación y agrupación en grandes áreas temáticas de los temas planteados por quienes participan en una "conferencia de consenso". En una primera etapa se dispondrían grandes tablones, uno por cada uno de las áreas temáticas identificadas, para recoger, mediante notas o métodos similares, críticas, proposiciones y conclusiones intermedias de los participantes. En una segunda etapa se haría un análisis de contenido interpretativo sobre estas aportaciones. En la tercera etapa se realizaría una reunión de discusión, para llegar a un consenso, con transcripción de la misma. En una cuarta etapa ser haría una consulta sobre el documento resultante de la discusión anterior y en una quinta y última etapa se votarían las propuestas de éstas.

Como podemos ver, se trata de una metodología cuya filosofía es cercana a los "foros híbridos" (fórums hybrides) de Barthes, Callon y Lascoumes (2014), y aunque desde esta metodología podrían seguirse utilizando herramientas como los cuadros de mandos integrales el enfoque filosófico y metodológico subyacente sería considerablemente distinto. Mientras que, como ya vimos anteriormente, la filosofía del cuadro de mandos integral, tal y como suele desarrollarse, establece a priori relaciones causales que permiten identificar los aspectos claves en los que una organización se ha de centrar para conseguir sus objetivos, desde este enfoque las relaciones causales no se pueden establecer a priori. Esto se relaciona con el diferente papel que se asignaría al conocimiento de expertos y profanos en la gestión de las realidades concretas. En los ámbitos del conocimiento científico- técnico el conocimiento del experto sobre una materia es cualitativa y cuantitativamente distinto al del profano: tendemos a pensar que un investigador en física de materiales sabe más del tema de lo que sabe el director de una fábrica de vigas. Por el contrario, en los ámbitos que se acercan al terreno de lo socio- político, cabe hablar más de diferencias cualitativas que cuantitativas en el conocimiento de los distintos agentes implicados. En cierto sentido, más que decir que el conocimiento que tiene un académico sobre el turismo es mayor que el que puede tener un director de hotel o un recepcionista, lo que tendemos a pensar es que se trata de un conocimiento distinto.

Por lo tanto, aunque esto no tiene que ser necesariamente incompatible con la filosofía original de los cuadros de mandos, pero sí con cómo ésta se ha acabado concretando, el papel de los agentes implicados en la formulación de las cuestiones claves (Key Performance Indicators) es muy distinto según se parta de una filosofía más "socio- participativa" o más "científico- tecnocrática". Desde una perspectiva tecnocrática, el papel de quien ayuda a elaborar un cuadro de mandos (normalmente, 
consultor, experto) es indicar a la organización cuáles son las variables clave: por poner sólo algunos ejemplos, pernoctaciones, Revpar, o porcentaje de aguas que se recicla. Por el contrario, desde una perspectiva socio- participativa, el papel de quien ayuda a elaborar un instrumento de medición de la actividad turística sería el de facilitador, que ayuda a los miembros de una organización o grupo a identificar lo que para ellos es importante, y a llegar a acuerdos y compromisos (consensos) entre lo que es importante para unos grupos y para otros. En el primero de los casos "un grupo humano (organización) paga al experto para que le diga lo que es importante". En el segundo de los casos, "un grupo humano (organización) paga al experto para que le ayude a identificar lo que para ellos es importante".

\section{CONCLUSIONES: CIENCIA Y POLÍTICA EN LA PLANIFICACIÓN TURÍSTICA}

El objetivo de este trabajo era analizar la extensión de herramientas derivadas de la planificación estratégica, como los cuadros de mando, y cuadros de mando integrales, a la gestión de los destinos turísticos. Para ello hemos considerado en primer lugar cómo han surgido estos conceptos, en el campo de la gestión empresarial a finales del siglo XX. Posteriormente hemos puesto los conceptos en el marco más amplio de la disciplina de la planificación estratégica, que como hemos visto puede entenderse desde una visión más humanista o bien desde una visión más tecnocrática, que es la que predomina, y que contribuye a dotar de legitimidad a las decisiones tomadas en base a herramientas como el cuadro de mandos, en la medida en que se presentan como decisiones basadas en un análisis objetivo y "científico" de la realidad, como medidas fundamentalmente técnico y sin connotaciones políticas. Posteriormente hemos analizado la literatura sobre el uso de cuadros de mando al sector turístico. Aunque el uso de instrumentos de medición para la gestión estratégica en el sector turístico está relativamente extendido (véase Sainaghi, Phillips y Zavarrone, 2017), es necesario destacar que si bien este tipo de herramientas parecen poder aplicarse de manera poco problemática al turismo cuando se hacen a nivel de empresa, surgen problemas metodológicos y filosóficos, relacionados en último término con la gobernanza, cuando se intentan aplicar a la gestión de los destinos turísticos, y de las entidades supuestamente encargadas de la misma, las Organizaciones de Gestión de Destinos (DMOs, por las siglas en inglés de "Destination Management Organizations"). La idea de planificación estratégica implica la determinación de cuáles han de ser los objetivos a perseguir y el establecimiento de un sistema de indicadores para ver en qué medida la gestión está logrando aquellos objetivos que se pretendía alcanzar. En entes tan complejos socialmente como lo son los destinos turísticos, aplicar la perspectiva de la planificación estratégica implica asumir distintos supuestos.

En primer lugar, el desarrollo de un sistema de indicadores para la toma de decisiones implica el supuesto de que existe un único agente, individual o colectivo, que toma las decisiones. Pero el hecho es que la realidad de los destinos turísticos desmiente este supuesto. Así, por ejemplo, ¿quién toma decisiones sobre la ordenación del territorio de costa? En la costa de un municipio turístico de Canarias, por ejemplo, toman decisiones, sin salir del ámbito de lo público, desde el Gobierno de España al Ayuntamiento, pasando por el Cabildo y la Administración Autonómica. El segundo supuesto que es necesario asumir para hablar en propiedad de planificación estratégica es que la toma de decisiones se puede basar en objetivos consensuados entre los distintos agentes que intervienen, lo que parece inspirado en una visión armónica y 
orgánica de la sociedad, en la que caben lemas del tipo "lo que es bueno para unos es bueno para todos". La gestión cotidiana de los destinos turísticos desmiente este supuesto: la gestión de los destinos es una arena para el conflicto entre distintos agentes con intereses diversos, en unos casos coincidentes, en otros complementarios y en otros contrapuestos, de manera que en la práctica más que de consensos de lo que se trata es de alcanzar compromisos razonables. Por ello es necesario incorporar el concepto de gobernanza para comprender la gestión de los destinos turísticos, analizando los distintos recursos que ponen en acción agentes diversos para imponer su visión de la realidad. Y es que la principal aportación que el desarrollo de Cuadros de Mandos Integrales hace a la gestión de los destinos turísticos pasa, precisamente, por su aportación a la gobernanza. Una DMO (Organización de Gestión de Destinos Turísticos) que pretenda operacionalizar su estrategia a través de un Cuadro de Mandos Integral, deberá someterse a un importante proceso interno de búsqueda de acuerdos, consensos y/o compromisos para establecer cuáles son los objetivos que se pretenden alcanzar, para establecer cómo pueden medirse estos objetivos y cómo corregir las posibles desviaciones. Sin embargo, como a menudo se cae en el fetichismo de la técnica, pensando que ésta es eficaz per se, y no por el proceso que implica su elaboración, no es extraño que desde entidades públicas como pueden ser las Consejerías de Turismo, se pida la elaboración de Cuadros de Mando para poder tomar mejores decisiones, como si el que una decisión fuera "mejor" o "peor" fuera algo absoluto que no dependiera de los objetivos que se pretenden lograr. Por ello a menudo se pretende utilizar este tipo de técnicas para tratar de zanjar de manera "técnica" lo que son siempre cuestiones políticas, del tipo de ¿cuál es el número de turistas que consideramos deseable? ¿Cuál la ratio entre turistas y residentes que nos parece más adecuada? ¿Cuál es el nivel de impacto ecológico que se debe evitar alcanzar? A menudo parece plantearse que el cuadro de mandos ha de orientar la elaboración de la política turística, cuando si entendemos en qué consiste el concepto debería ser justamente al revés: es la política lo único que puede dar sentido a la elaboración de cuadros de mandos.

Así que, a modo de conclusión, este el trabajo realizado nos ha permitido ver que, si bien el desarrollo de cuadros de mando y sistemas de indicadores para la gestión de los destinos turísticos se hace, supuestamente, con el objetivo de encontrar las medidas políticas que mejor "convengan" a estos, en la práctica se acaban convirtiendo en una herramienta, más que para extraer información, para inyectar información. Términos y filosofías de la planificación estratégica y de los Cuadros de Mando Integral han impregnado la práctica cotidiana de administraciones públicas y empresas y han acabado teniendo un efecto performativo, ya que para poder optar a ayudas y subvenciones muchas organizaciones han de utilizar términos y conceptos propios de esta literatura, por ejemplo, identificando medidas fácilmente cuantificables de cumplimiento de objetivos, en la línea de los denominados KPI (Key Performance Indicators). En la Administración Pública el uso de CMI se ha convertido en una práctica tan común que a menudo se tiende a concebir su elaboración como el objetivo del trabajo de los staff técnicos y de apoyo de un determinado campo o sector como instrumento de toma de decisiones de los políticos. Y, en último término, este tipo de instrumentos acaban sirviendo, a menudo, para imponer medidas políticas como si fueran medidas técnicas, contribuyendo a crear consensos (o a imponerlos) acerca de cuál ha de ser la dirección que lleve la gestión de un destino turístico. Así, por ejemplo, utilizar como indicador "empleos directos creados por el turismo" es una medida aparentemente técnica pero que tiene implicaciones políticas, ya que acaba favoreciendo al sector alojativo hotelero frente al extrahotelero. En cualquier caso, creemos que el 
desarrollo de metodologías para la elaboración de cuadros de mando integrales en el sector turístico, en el sentido de lo desarrollado en el llamado "Consenso de St. Gallen" (véase Laesser y Beritelli, 2013) parecen tener amplias potencialidades, en la medida en que permitan mejorar la gobernanza de los destinos turísticos. En cualquier caso, como todo trabajo, el que aquí hemos realizado tiene sus limitaciones, que indican también líneas interesantes para investigaciones futuras. Al respecto destacaremos que los dos enfoques predominantes de la planificación estratégica, el que hemos denominado tecnocrático, ejemplificado en normativas internacionales como las de la OMT para la medición de la sostenibilidad (véase WTO, 2005) y el que hemos denominado más humanístico, ejemplificado en la idea del "Consenso de St. Gallen" se han aplicado, respectivamente, a nivel macro y micro. Posiblemente uno de los mayores desafíos de cara al futuro sea integrar estos dos niveles, ayudando a construir estructuras de gobernanza que permitan que la gestión de los destinos turísticos favorezca más a los agentes implicados, repercutiendo todo ello, en último término, en un impacto más positivo de la actividad turística en los lugares en que se desarrolla.

\section{BIBLIOGRAFÍA}

BALLVÉ, A. M. Creando conocimiento en las organizaciones con el Cuadro de Mando Integral y el Tablero de Control. En Revista de Contabilidad y Dirección. 2006, Vol. 3, , pp. 13-38

BARTHE, Y., CALLON, M y LASCOUMES, P. Agir dans un monde incertain. Essai sur la démocratie technique. París, Le Seuil, 2014.

BLANCO, F. El control integrado de gestión, Limusa, México,1976.

BOLTANSKI, L. y CHIAPELLO, E. El nuevo espíritu del capitalismo. Madrid: Ediciones Akal, 2002.

CALLON, M. Performativity, misfires and politics. En Journal of Cultural Economy, 2010, vol. 3, no 2, p. 163-169.

DÁVILA, A. El cuadro de mando integral. Revista de antiguos alumnos IESE, 1999.

EARLE, J. MORAN, C. y WARD-PERKINS, Z. Econocracy. Manchester:Manchester University Press, 2016.

ESCOBAR RODRÍGUEZ, T. El papel del cuadro de mando en la gestión estratégica de la empresa. Revista Española de Financiación y Contabilidad, 1999, p. 1075-1099.

FERNÁNDEZ RODRÍGUEZ, C. J.El discurso del management: tiempo y narración. Madrid: Centro de Investigaciones Sociológicas, 2007.

GIGERENZER, G. y SELTEN, R. (ed.). Bounded rationality: The adaptive toolbox. Cambridge: Massachussets Institute of Technology Press, 2002.

GRANER, L. De la contabilidad de costes al tableau de bord. Barcelona: Marcombo Boixareu Editores, 1991.

GREY, $\mathrm{C}$ y WILlMOTT, $\mathrm{H}$ (ed.). Critical management studies: A reader. Oxford:Oxford University Press, 2005.

KAHNEMAN, D. Maps of bounded rationality: Psychology for behavioral economics. American economic review, 2003, vol. 93, no 5, p. 1449-1475. 
KAPLAN, R. y NORTON, D. The balanced scorecard: measures that drive performance. En Harvard Business Review, v. 70, n. 1, p. 71-79.

KAPLAN, R. y NORTON, D. Translating Strategy into Action: the balanced scorecard.Boston: Harvard Business School Press, 1996

KAPLAN, R. S. y NORTON, D. P. El cuadro de mando integral. Barcelona: Gestión, 2000

KAPLAN, R.; NORTON, D. (2004): Strategy maps: Converting intangible assets into tangible outcomes. Boston: Harvard Business School Press, 2004

KUHN, S. T. La estructura de las revoluciones científicas. Buenos Aires: Fondo de cultura económica, 2004 (la versión original es en inglés, de 1962).

LATOUR, B. Ciencia en acción: cómo seguir los científicos e ingenieros a través de la sociedad. Barcelona: Labor, 1992.

LAUZEL, P., CIBERT, A. yCARRIÓ, J. V. De los ratios al cuadro de mando: cómo se estructuran y utilizan estos medios de gestión. Francisco Casanovas, 1967.

LAESSER, C y BERITELLI, P. St. Gallen consensus on destination management. Journal of Destination Marketing \& Management, 2013, vol. 2, no 1, p. 46-49.

MEYER, J. Le contrôle de gestion, París: Presses Universitaires de France, 1969.

MINTZBERG, H. Managers not MBA. San Francisco: Berret-Koeler.

MORA, A. y VIVAS, C. Nuevas herramientas de gestión pública: el cuadro de mando integral. Madrid: AECA, 2001.

REINHOLD, S., LAESSER, C., y BERITELLI, P. 2014 St. Gallen Consensus on destination management. Journal of Destination Marketing \& Management, 2015, vol. 4, no 2, p. 137-142.

RUSSO, J. Balanced scorecard versus tableau de bord. Revista da Câmara dos Técnicos Oficiais de Contas, n. 62, p. 56-63, 2005.

SAINAGHI, R. PHILLIPS, P y ZAVARRONE, E. Performance measurement in tourism firms: A content analytical meta-approach. En Tourism Management, 2017, vol. 59, p. 36-56.

SANTOS, M.; FIDALGO, E. El balanced scorecard o cuadro de mando integral y el cuadro de mando tradicional: principales diferencias. Técnica Contable, n. 673, p. 13$17,2005$.

SENNETT, R. La cultura del nuevo capitalismo, Barcelona, Anagrama, 2009

SILVER, N. The signal and the noise. The Art and Science of Prediction, Londres, Penguin Books, 2012

SIMON, H. Bounded rationality and organizational learning. Organization science, 1991, vol. 2, no 1, p. 125-134.

TORRES DELGADO, A. Y LÓPEZ PALOMEQUE, F. Measuring sustainable tourism at the municipal level, en Annals of Tourism Research, 2014, nº 49, 122- 137

TORRES LORENZO, y HERNÁNDEZ MARTÍN, R. Propuesta para el diseño de un sistema de indicadores integrado para la coyuntura turística de Canarias. Cuadernos de Estudios Empresariales, 2013, vol. 23, p. 55. 
VILA, M, COSTA, G y ROVIRA, X: The creation and use of scorecards in tourism planning: a Spanish example, En Tourism Management, 2010, nº 31, 232-239.

UNEP y UNWTO: Making tourism more sustainable. A guide for policy makers. Paris: United Nations Environment Programme, Division of Technology, Industry and Economics y Madrid, United Nations World Tourism Organization. 2005 